\title{
Predictions Specify Reactive Control of Individual Digits in Manipulation
}

\author{
Yukari Ohki, Benoni B. Edin, and Roland S. Johansson \\ Section of Physiology, Department of Integrative Medical Biology, Umeå University, S-901 87 Umeå, Sweden
}

\begin{abstract}
When humans proactively manipulate objects, the applied fingertip forces primarily depend on feedforward, predictive neural control mechanisms that depend on internal representations of the physical properties of the objects. Here we investigate whether predictions of object properties also control fingertip forces that subjects generate reactively. We analyzed fingertip forces reactively supporting grasp stability in a restraining task that engaged two fingers. Each finger contacted a plate mounted on a separate torque motor, and, at unpredictable times, both plates were loaded simultaneously with forces tangential to the plates or just one of the plates was loaded. Thus, the apparatus acted as though the plates were mechanically linked or as though they were two independent objects. In different test series, each with a predominant behavior of the apparatus and with interspersed catch trials, we showed that the reactive responses clearly reflected the predominant be-
\end{abstract}

havior of the apparatus. Whether subject performed the task with one hand or bimanually, appropriate reactive fingertip forces developed when predominantly both contact plates were loaded or just one of the plates was loaded. When a finger was unexpectedly loaded during a catch trial, a weak initial reactive response was triggered, but the effective force development was delayed by $\sim 100 \mathrm{msec}$. We conclude that the predicted physical properties of an object not only control fingertip forces during proactive but also in reactive manipulative tasks. Specifically, the automatic reactive responses reflect predictions at the level of individual digits as to the mechanical linkage of items contacted by the fingertips in manipulation.

Key words: manipulation; human hand; fingertip forces; internal models; sensorimotor prediction; grasp stability; reactive responses
The sensorimotor control of the hand during object manipulation is characterized by feedforward, predictive control policies that reflect internal models of the relevant physical properties of the objects (Johansson and Cole, 1994; Johansson, 1996, 1998; Wing, 1996). Predictions of the friction between the object and individual fingertips determine, for instance, the ratio between forces applied normal and tangential to the contact surfaces of an object (Johansson and Westling, 1984; Edin et al., 1992; Burstedt et al., 1997, 1999). Moreover, when the subject's own movements cause destabilizing tangential load forces, anticipatory grip forces are generated normal to the contact surface to prevent object slippage (Johansson and Westling, 1984, 1988b; Flanagan et al., 1993; Flanagan and Tresilian, 1994; Blakemore et al., 1998). The fingertip forces predict the mass and mass distribution of the objects (Johansson and Westling, 1988a; Goodwin et al., 1998; Wing and Lederman, 1998; Johansson et al., 1999) and also their shape (Jenmalm and Johansson, 1997; Goodwin et al., 1998) and more complex loads that result from the viscous and spring properties of the objects (Flanagan and Wing, 1997). These grip actions are based on predictions of the consequences of self-generated actions, and this is essential given the inevitable neuromechanical delays that curtail the usefulness of closed-loop feedback control (Hogan et al., 1987; Johansson and Cole, 1994; Johansson, 1998).

\footnotetext{
Received July 11, 2001; revised Oct. 4, 2001; accepted Oct. 30, 2001.

This study was supported by Swedish Medical Research Council Project 08667, the Göran Gustafsson Foundation for Research in Natural Sciences and Medicine, and the 5th Framework Program of the European Union Project QLG3-CT-1999-00448.

Correspondence should be addressed to Dr. Yukari Ohki at her present address: Kyorin University School of Medicine Department of Physiology 6-20-2 Shinkawa, Mitaka-shi, Tokyo 181-8611, Japan. E-mail: ohkiy@kyorin-u.ac.jp.

Copyright (C) 2002 Society for Neuroscience $0270-6474 / 02 / 220600-11 \$ 15.00 / 0$
}

Predictions have also been demonstrated in reactive tasks when subjects restrain "active" objects from moving. The simplest expression of such predictions is an increased background normal force that provides an increased safety margin against slips when subjects expect the tangential load to rapidly increase some time in the future (Johansson and Westling, 1988b; Johansson et al., 1992a,b; Cole and Johansson, 1993; Winstein et al., 1999). However, the automatic normal force responses (minimum latencies of $60-80 \mathrm{msec}$ ) that are triggered by cutaneous receptors in the fingertips (Johansson et al., 1992b,c; Macefield et al., 1996) reveal that the sensorimotor system in humans also attempts to predict the future behavior of active objects by scaling the responses by early cues about the rate of the load force changes (Cole and Abbs, 1988; Johansson et al., 1992b) and friction (Cole and Johansson, 1993; Birznieks et al., 1998).

In self-paced, proactive bimanual tasks, the expression of anticipatory grip actions depends on whether a test apparatus behaves like one or two physical objects (Blakemore et al., 1998). To investigate whether the sensorimotor mechanisms that mediate reactive grip responses also express predictions in a similar context, we developed a task that engaged two fingers, each in contact with a plate mounted on a separate torque motor. In "linked" trials, both plates were simultaneously loaded, and the apparatus behaved like a single physical object held by the two fingers; in "unlinked" trials, load force was generated at only one of the contact plates at a time, and the apparatus accordingly simulated two independent objects. By using different series, each with a predominant object behavior and with interspersed catch trials, we assessed whether the sensorimotor transformations underlying the reactive responses at the level of individual digits were modified as a function of the behavior of the apparatus. 

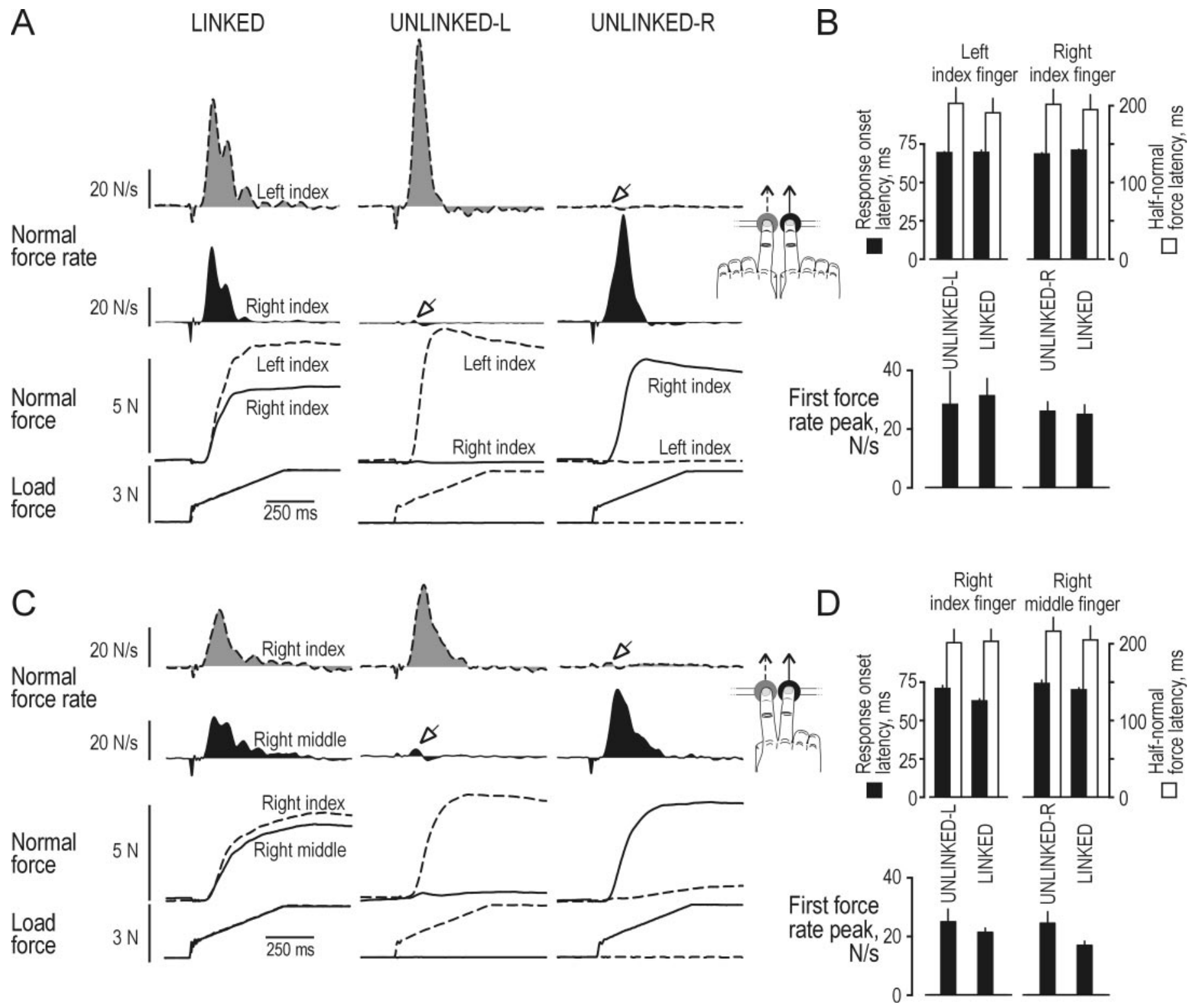

Figure 1. Responses to the predominant behavior of the apparatus. $A, C$, Averaged normal force rate and normal and load force in a single subject when the two fingers were predominantly loaded concurrently (LINKED) or singly (UNLINKED-L for left finger and UNLINKED-R for right finger). Dashed curves and gray shading indicate data for the left finger (left index finger in $A$ and right index finger in $C$ ), and solid curves and black shading indicate data for the right finger (right index finger in $A$ and right middle finger in $C$ ). Arrows indicate responses in fingers that were typically not loaded in the UNLINKED series. $B, D$, Height of bars gives mean values of the following response measures for loaded fingers: response onset latency ( filled bars), half-normal force latency (open bars), and first force rate peak. The filled and open bars are linked to indicate that they refer to the same test series. Data are averaged across all subjects; error bars indicate unilaterally one SEM $(n=7)$. These response measures were similar regardless of grasp configuration and test series. $A, B$, Bimanual grasp. $C, D$, Unimanual grasp.

\section{MATERIALS AND METHODS}

With the approval of the local ethical committee, experiments were performed on two healthy female and five male right-handed human volunteers of ages between 23 and 53 years, all who gave their informed consent. The general procedure and apparatus was the same as described in a previous study (Ohki and Johansson, 1999). Briefly, the subjects were seated with the forearms extended anteriorly and supported by a tabletop up to the palms. With the palms down, the subjects used the tips of two fingers, positioned side-by-side, to restrain an instrumented manipulandum (Fig. 1, insets). Two grasp configurations were used: the two fingers were either the right and left index fingers (bimanual grasp) or the right index and middle fingers (unimanual grasp). For each digit, the manipulandum had a horizontally oriented flat contact plate covered by suede (diameter of $30 \mathrm{~mm}$; center-to-center distance of $32 \mathrm{~mm}$ ). The contact plates could be loaded independently in the distal direction by force servos $(0-10 \mathrm{~N}, 0-15 \mathrm{~Hz}$ bandwidth; noise of $<0.05 \mathrm{~N})$. The grip and load forces applied by the fingertips were measured perpendicular and tangential to the surfaces of the plates, respectively. The position of each plate was transduced to a resolution of $0.05 \mathrm{~mm}$, and the grasp plates were servo-regulated to constant position (stiffness of $1.2 \mathrm{~N} / \mathrm{mm}$ ) when the fingers were not touching the manipulandum. The subjects were blindfolded during the experiments, and the apparatus provided no sound cues.

Behavior of the apparatus. We used four test series, each with a predominant behavior of the apparatus (Table 1). The sequence of presentation of the various test series was randomized across the subjects. In each of the test series, one type of trials was dominant, i.e., occurred with the highest probability. In one of the test series ("LINKED"), loads were most commonly applied simultaneously to both contact plates, whereas during single trials in the other three test 
Table 1. The four test series used

\begin{tabular}{|c|c|c|c|c|c|c|}
\hline \multirow[b]{2}{*}{ Test series } & \multicolumn{4}{|c|}{ Actual behavior of the apparatus } & \multicolumn{2}{|c|}{$\begin{array}{l}\text { Loading } \\
\text { probability }\end{array}$} \\
\hline & $\begin{array}{l}\text { Trials } \\
(n)\end{array}$ & $\begin{array}{l}\text { Both } \\
\text { plates } \\
\text { loaded }\end{array}$ & $\begin{array}{l}\text { Only } \\
\text { left } \\
\text { plate } \\
\text { loaded }\end{array}$ & $\begin{array}{l}\text { Only } \\
\text { right } \\
\text { plate } \\
\text { loaded }\end{array}$ & $\begin{array}{l}\text { Right } \\
\text { plate }\end{array}$ & $\begin{array}{l}\text { Left } \\
\text { plate }\end{array}$ \\
\hline $\begin{array}{l}\text { LINKED (both } \\
\text { plates) }\end{array}$ & 55 & $86 \%$ & $7 \%$ & $7 \%$ & $93 \%$ & $93 \%$ \\
\hline $\begin{array}{l}\text { UNLINKED-L } \\
\text { (left plate) }\end{array}$ & 40 & $10 \%$ & $80 \%$ & $10 \%$ & $20 \%$ & $90 \%$ \\
\hline $\begin{array}{l}\text { UNLINKED-R } \\
\text { (right plate) }\end{array}$ & 40 & $10 \%$ & $10 \%$ & $80 \%$ & $90 \%$ & $20 \%$ \\
\hline $\begin{array}{l}\text { UNLINKED-LR } \\
\text { (alternating } \\
\text { plates) }\end{array}$ & 34 & $12 \%$ & $44 \%$ & $44 \%$ & $56 \%$ & $56 \%$ \\
\hline
\end{tabular}

series, the load was typically applied to either the left or the right contact plate ("UNLINKED"). Hence, a given digit was subjected to one of three loading conditions: loaded concurrently with its partner finger, loaded separately, or not loaded at all. The term "catch trial" is used for nonstandard trials during the test series, e.g., a trial when a single digit was loaded in a LINKED test series; these trials served to probe for response preparation based on subjects' predictions of the behavior of the apparatus.

The LINKED test series consisted of 55 trials. During the last 37 linked trials, eight unlinked catch trials were randomly interspersed, four with only the left plate loaded and four with only the right plate loaded. Thus, the two contact plates were linked in $86 \%$ (47 of 55) of the trials, and the probability of loading of each finger was $93 \%$. The same test series was used in a previous study (Ohki and Johansson, 1999).

In the UNLINKED test series, the load was applied predominantly to either the left ("UNLINKED-L") or the right ("UNLINKED-R") contact plate, or the left and right contact plates were loaded with equal probability ("UNLINKED-LR"). In the UNLINKED-L and UNLINKED-R series, the left and right contact plate, respectively, was loaded in 32 trials, whereas the other plate was loaded in four trials and both loaded in four trials. The eight catch trials were randomly interspersed among the last 29 trials. Thus, in $80 \%$ (32 of 40) of the trials, only one plate was loaded, but the total probability of loading of that plate was $90 \%$ and that of the other plate was $20 \%$. During the UNLINKED-LR test series, either the right or the left plate was loaded in a random order and with equal probability (15 trials each). Four linked catch trials were interspersed randomly during the last 20 trials of the series, i.e., 4 of 34 $(12 \%)$ of the trials were linked and the total probability of loading either plate was $\sim 56 \%$. In contrast to the other three test series, the predominant behavior of the apparatus during the UNLINKED-LR test series thus did not allow useful predictions as to which plate would be loaded.

Load characteristics. The load was superimposed on a $0.2 \mathrm{~N}$ constant baseline load at each contact plate; the baseline load was automatically applied when the subject contacted the contact plate. The load comprised a load phase (phase of distal load force increase), followed by a hold phase and a rapid unloading. During the first $20 \mathrm{msec}$ of the load phase, the load increased abruptly by $0.8 \mathrm{~N}$, and, after this "load step," it continued to increase at a constant rate of $4 \mathrm{~N} / \mathrm{sec}$ for $0.5 \mathrm{sec}$ to the hold phase that was maintained for $1.0 \mathrm{sec}$ (Fig. 1, Load force traces); the load at each digit during the hold phase was thus $3 \mathrm{~N}$. The high initial load force rate served to trigger a distinct normal force response, and the following load ramp increase during the load phase guaranteed substantial response amplitude (cf. Johansson et al., 1992b; Ohki and Johansson, 1999). The interval between consecutive load trials was randomized between 1.5 and $2.5 \mathrm{sec}$.

Instructions to subjects. Subjects were instructed to simply restrain the plates from moving. They were neither suggested useful strategies nor were they given instructions regarding suitable normal forces. To make it easier for the subjects to learn the nature of each test series, the experimenter informed the subjects about the three possible behaviors of the apparatus and the one of these that was predominant in the upcoming test series and would occur in nearly all trials. In addition, the structure of the series allowed consistent early experiences of the prevailing predominant behavior of the apparatus, i.e., the catch trials occurred during the later part of the series.

Data collection and analysis. Data were collected and analyzed with a laboratory computer system (SC/ZOOM; Section of Physiology, Integrative Medical Biology, University of Umeå). The force signals were sampled at $400 \mathrm{~Hz}$, and the position signals were sampled at $100 \mathrm{~Hz}(12$ bit resolution). Event markers related to onsets of the various phases of each load trial were sampled, as well ( $0.1 \mathrm{msec}$ resolution). Force rates were obtained as a function of time by symmetrical numerical time differentiation within a time window corresponding to \pm 5 data samples.

The following measures were taken from individual trials. The response onset latency was the time interval from the onset of the load force increase to the onset of the reactive normal force increase; the onset of the reactive normal force increase was assessed by defining a threshold force rate. During the force increase, we identified one or more peaks in the normal force rate profile (for details, see Ohki and Johansson, 1999). We measured the time of occurrence and the amplitude for the first peak. We also measured the time when the normal force had increased halfway from the pretrial normal force value (the normal force at the onset of the load increase) to the value reached at the end of the load phase; the half-normal force latency was the interval between the onset of load increase and this point and indicates speed of effective force development. The response amplitude was measured as the difference between the normal force measured $0.05 \mathrm{sec}$ before the end of the hold phase and the pretrial normal force. Concerning response parameters pertaining to latencies and force rate peaks, measurements were obtained only when maximum normal force rate exceeded $5 \mathrm{~N} / \mathrm{sec}$; weaker responses could not be reliably detected in single trial records. For trials in which a force response was not present according to this criterion, no latency measurements were obtained, and the peak rates were set to zero in the statistical analyses for these trials. This occurred only at a nonloaded finger in a minority of the trials in test series in which that finger was loaded only in catch trials (see Results). Otherwise, all measurements above were obtained also for nonloaded fingers. Responsiveness was calculated as the percentage of trials within a series with measurable force responses.

Numerical values of normal forces and tangential forces were transferred to a statistical program (Statistica; StatSoft Inc., Tulsa OK). The analysis was focused on the responses of individual digits during the various loading conditions and behaviors of the apparatus, and data from individual digits are presented in the illustration. In a previous study in which the apparatus simulated one object (predominantly LINKED trials), for a given grasp configuration there was, however, no difference between the digits (Ohki and Johansson, 1999). A preliminary analysis of the present data confirmed this. Accordingly, to simplify the statistical analysis, we pooled data from both digits engaged in each grasp configuration for each combination of loading conditions and behavior of the apparatus, i.e., data were pooled from the right and left index fingers of the bimanual grasp and from the right index and middle fingers of the unimanual grasp. However, to display the similarities between the digits, we chose to include data for both fingers in the figures. We analyzed effects of the predominant behavior of the apparatus on response intensity or time variables, and, unless otherwise stated, the statistical reports emanate from two-way repeated-measures ANOVAs whose factors were behavior of the apparatus (three levels: LINKED, UNLINKED-L, and UNLINKED-R, i.e., excluding the UNLINKED-LR test series) and grasp configuration (two levels: bimanual and unimanual). We separately compared responses between UNLINKED-LR and LINKED series; thus, factors for these ANOVAs included only two levels pertaining the behavior of the apparatus (UNLINKED-LR and LINKED) and two levels representing grasp configuration. In other ANOVAs, the loading condition (two levels; finger concurrently loaded in linked trials and loaded separately, or loaded separately and not loaded) was used as one factor. Planned comparisons were performed to analyze specific effects. In the statistical analysis of probability values, we took into account the probability distribution by standard logit transformation of the response variables. The level of probability selected as statistically significant was $p<0.05$, and, unless indicated otherwise, population estimates are presented as mean $\pm 1 \mathrm{SD}$. For each subject and for each of the experimental conditions, values from all trials were averaged, and the subject mean and SD were calculated for these "average trials" from seven subjects (in graphs, one standard-error of mean, SEM, is indicated unilaterally; $n=7)$. The average trials were also used in ANOVAs. 


\section{RESULTS}

Results are divided in three parts. In the first, we analyze normal force responses elicited automatically when the subjects restrained the contact plates from moving during trials with the predominant behavior of the apparatus. In particular, we investigate whether predictions of the behavior of the apparatus influenced the load-to-normal force sensorimotor transformations for individual digits when the apparatus acted as though the plates were mechanically linked (LINKED test series) versus representing two independent objects (UNLINKED test series). In the second part, we analyze the state of the corresponding sensorimotor transformations when the behavior of the apparatus was unpredictable (UNLINKED-LR test series). Finally, we address differences between the bimanual and unimanual grasp configurations concerning expressions of response preparations at the level of individual digits.

\section{Predictable behavior of the apparatus}

All subjects quickly adapted to the predominant behavior of the apparatus in all test series, i.e., in the LINKED test series, loading promptly elicited normal force responses in both digits, whereas in the UNLINKED-L and UNLINKED-R test series, loading resulted in a prompt normal force increase at the loaded digit (Fig. $1 A, C$ ). At most, meager responses were observed in the partner digit (Fig. $1 A, C$, arrows in Normal force rate traces). With the predominant behavior of the apparatus, the responsiveness, the response onset latency, the magnitude of the first peak force rate, and the half-normal force latency were quantitatively similar for the loaded digits, regardless of grasp configuration and test series (Fig. $1 B, D$ ). The responsiveness was always $100 \%$, and the grand mean of the response onset latency, the first peak force rate, and the half-normal force latency was $70 \pm 5 \mathrm{msec}, 25 \pm 12$ N/sec, and $202 \pm 49 \mathrm{msec}$, respectively.

The simplest explanation for these observations is, of course, that the elicited normal force response at a finger was stereotypically driven by afferents activated by the loading of the individual finger. This is a sufficient explanation only if the response to a particular type of loading was the same, regardless of the behavior of the apparatus, i.e., if the responses were the same in all test series when, for instance, only the left digit was loaded; this was, however, not the case. Catch trials were interspersed to reveal the sensorimotor settings of the subjects during the predominant condition of a test series. The catch trials demonstrate unequivocally that the observed responses at individual finger could not be completely explained as simple consequences of afferent activation operating on a fixed load-to-normal force sensorimotor transformation. Rather, the involved load-to-normal force sensorimotor transformations were prepared for a certain behavior of the apparatus.

\section{Responses to catch trials when a finger was unexpectedly loaded}

In two of the UNLINKED test series, the apparatus predominantly loaded a single finger, i.e., either the left (UNLINKED-L) or the right (UNLINKED-R) finger, whereas the partner digit remained unloaded. The loading condition during some of the catch trials of the UNLINKED test series was, however, the same as during the most common trials of the LINKED test series, i.e., both fingers were loaded concurrently. Figure 2 illustrates the normal force development at the two fingers during concurrent loading during the three test series for the bimanual $(A, B)$ and the unimanual $(C, D)$ grasp configuration. The phase plots (Fig. 2A,C) illustrate for linked trials the normal force at the finger contacting the left plate as a function of the normal force at the partner finger. If the afferent activation during concurrent loading synchronously triggered similar force responses at the two fingers, the normal forces should develop in parallel at the two fingers. That is, the curves in the phase plots of Figure 2, $A$ and $C$, should run close to the line of identity. Indeed, when the two fingers were concurrently loaded during the LINKED test series (Fig. 2, left column), the normal forces developed primarily in parallel at the two fingers, whereas this was clearly not the case in test series in which the left (Fig. 2, middle column) or the right (Fig. 2, right column) plate was predominantly loaded. In catch trials with concurrent loading in the UNLINKED-L test series, normal force development at the right finger typically lagged the force development at the left finger. Conversely, normal force development at the left finger typically lagged the right finger during the UNLINKED-R test series.

For any finger and under all conditions, when the finger was loaded in a trial of a test series during which the finger typically was not loaded, the amplitude of the first force rate peak (Newtons per second) was on average smaller than in other trials $\left(F_{(2,12)}=11.8 ; p<0.01\right)$, i.e., the elicited response was initially weaker (Fig. 3). Moreover, the response onset latency increased, but the effect was quantitatively small (Fig. 3) although statistically significant $\left(F_{(2,12)}=12.7 ; p<0.01\right)$. In the UNLINKED-L and UNLINKED-R test series, for instance, the onset of the response for a finger loaded infrequently occurred on average only $7 \pm 8 \mathrm{msec}$ later than that for the predominantly loaded partner finger (data pooled across all fingers). More importantly, when a finger typically not loaded was loaded, the time of effective force development gauged by the half-normal force latency was substantially delayed $\left(F_{(2,12)}=24.2 ; p<0.01\right)$ (Figs. 2, 3). The longest half-normal force latency $(285 \pm 44 \mathrm{msec})$ was observed for a finger infrequently loaded in the UNLINKED series, whereas the shortest half-normal force latencies were observed for a finger predominantly loaded in the UNLINKED series $(195 \pm 57 \mathrm{msec})$ and for both fingers in the LINKED test series $(197 \pm 50 \mathrm{msec}$ ) (Fig. 3). In the UNLINKED series, the half-normal force was reached on average $95 \pm 54 \mathrm{msec}$ later than that for the partner finger that was predominantly loaded (data from both grasp configurations pooled), whereas the absolute difference in latency of cooperating fingers was only $29 \pm 17 \mathrm{msec}$ in the LINKED test series. We interpreted that the delayed response in the unexpectedly loaded finger reflected the triggering of a corrective action to compensate for an erroneous prediction of the behavior of the apparatus.

The data analyzed above for the UNLINKED test series included all catch trials with concurrent loading of both fingers. As is apparent from the phase plane plots of the UNLINKED test series in Figure 2, however, a delayed response in the unexpectedly loaded finger did not appear in all catch trials, i.e., in a few trials the responses at the two fingers developed almost in parallel. To analyze the nature of overt corrective, delayed responses in more detail, trials in which the normal force rate exceeded $5 \mathrm{~N} / \mathrm{sec}$ later than $100 \mathrm{msec}$ were selected (in effect, the initial normal force response corresponding to first rate peak was ignored). From the unimanual and bimanual UNLINKED test series, 91 and $48 \%$ of the trials, respectively, were selected using this criterion (two subjects did not show any corrective response in some test series). On average, the corrective response appeared $274 \pm 55 \mathrm{msec}$ after the onset of load force increase and $168 \pm 60 \mathrm{msec}$ after the onset of the normal force response; the 


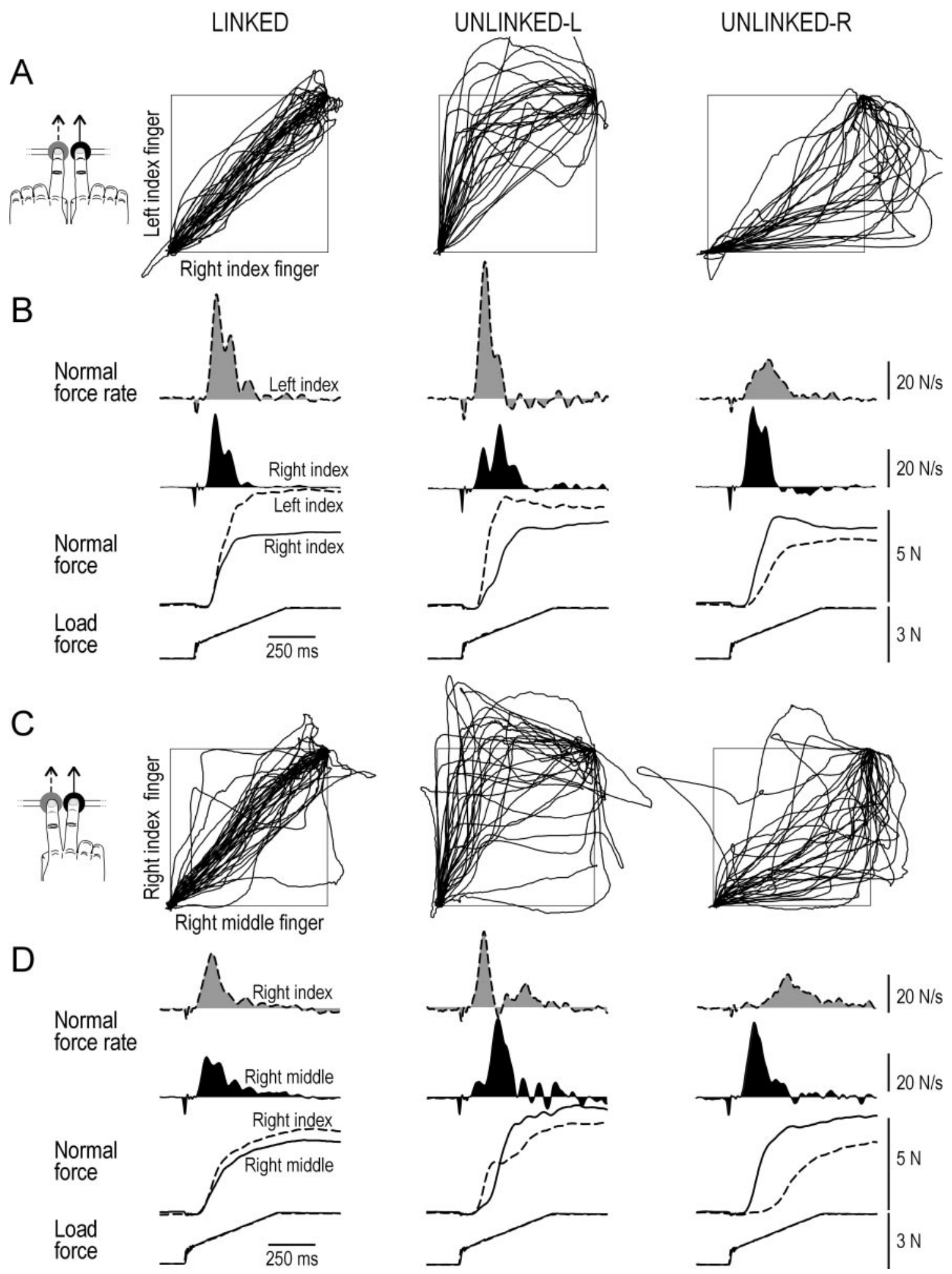

Figure 2. Responses to unexpected loading. $A, C$, Phase plots illustrating synchronization between fingers of normal force increases during the load phase for single trials with concurrent loading of both fingers. Normal force at the finger contacting the left plate is plotted against the normal force at the finger contacting the right plate. The force increase during the load phase was normalized for each finger to the values measured at the start and end of the load phase; the curves representing single trials are anchored at the bottom left and top right corners of the square, which thus represent the values measured at the start and end of the load phase. All trials by all subjects are superimposed. $B, D$, Normal force rates, normal forces, and load forces averaged across all trials with concurrent loading of the pair of digits within the respective test series performed by a single subject. For additional explanation, see caption of Figure 1, $A$ and C. $A-D$, Data for the LINKED test series represents responses to the predominant behavior of the apparatus, whereas data labeled UNLINKED-L and UNLINKED-R represent exceptional catch trials during these test series. The averaged traces and the phase plots show that functionally important normal force responses at the finger not expected to be loaded were delayed in the UNLINKED test series. $A$, $B$, Bimanual grasp. $C, D$, Unimanual grasp. 


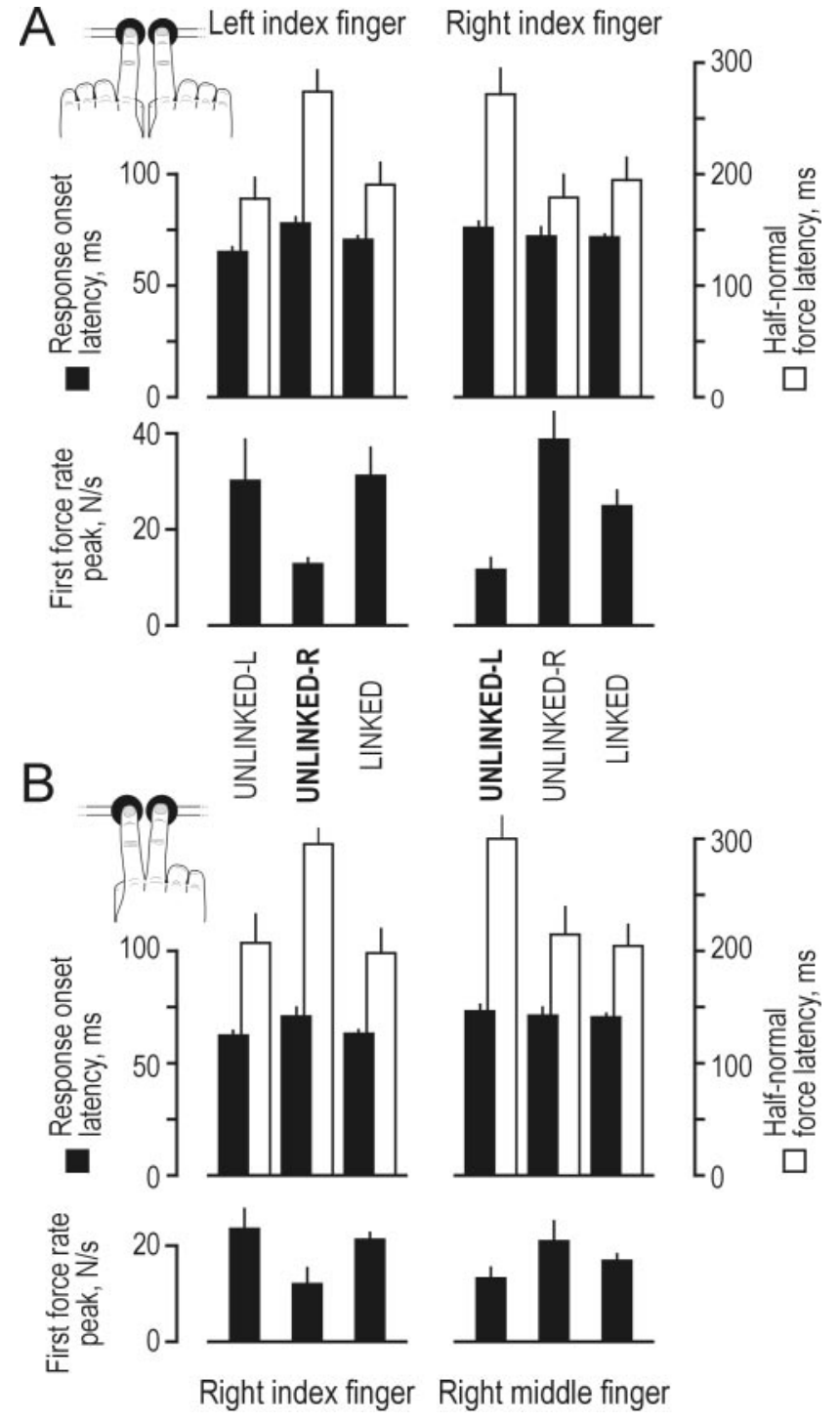

Figure 3. Responses in unexpectedly versus typically loaded fingers. Averaged response onset latency ( filled bars), half-normal force latency (open bars), and amplitude of first force rate peak for each finger grouped by test series during bimanual grasp $(A)$ and unimanual grasp $(B)$. The first force rate peak was significantly smaller and the half-normal force latency was significantly delayed in UNLINKED test series when a finger was unexpectedly loaded during catch-trials (test series labeled in bold) compared with both the LINKED test series and the UNLINKED test series in which the finger was typically loaded. Data were averaged across all subjects; error bars indicate unilaterally one SEM $(n=7)$.

latency of half-normal force increase was on average $297 \pm 64$ msec after the onset of loading (mean \pm SD; data from both grasp configurations pooled).

The predicted behavior of the apparatus thus profoundly influenced the subjects' reactive responses to loading of a finger, i.e., a similar afferent inflow elicited by the tangential force increase at a digit released automatic motor commands that were tailored to the predominant behavior of the apparatus. In contrast to the fast and resolute response generated at a finger expected to be loaded when a finger was unexpectedly loaded, subjects often generated a weak initial response, followed by a corrective response that appeared after a substantial delay after the onset of the load force increase.
Responses to catch trials when a finger was unexpectedly not loaded: interdigital reflexes

The observed response preparation suggested that the subjects prepared digit-specific sensorimotor transforms for a predicted behavior of the apparatus: a prompt response was prepared only for a digit likely to be loaded. However, interdigital reflexes could have contributed to the normal force responses (Ohki and Johansson, 1999), and their articulation could have depended on predictions concerning the behavior of the apparatus. Accordingly, we hypothesized that interdigital reflex facilitation between the fingers should be low in the UNLINKED test series, i.e., when the apparatus in effect simulated two objects, because the brain would individuate the control of the fingers. In contrast, when the apparatus simulated a single object (LINKED test series), there would be less need for individuating the control, and it seemed more likely that neural networks supporting facilitatory interactions between the sensorimotor controls of the fingers could be active. By analyzing normal force responses evoked at a finger unexpectedly not loaded, we exposed pure sensorimotor interactions between fingers; only sensory input associated with the loading of the partner finger could account for such responses, at least during the bimanual grasp.

Figure 4, $A$ and $C$, shows averaged responses from a single subject when one of the fingers were unexpectedly not loaded during the trials. The left column represents the responses when the right but not the left finger was loaded in the LINKED test series, i.e., test series in which the fingers were concurrently loaded in the majority of the trials. In these trials, afferent inputs originating from loading of the right finger must have driven the responses of the nonloaded left finger. As demonstrated previously (Ohki and Johansson, 1999), a single brief force rate peak dominated the response of a nonloaded finger in these catch trials. This peak corresponds to the first force rate peak of the loaded finger (in single trials, there were generally two or sometimes more peaks in the normal force rate profile). The middle and right columns of Figure 4, $A$ and $C$, illustrate analogous results when the left or the right finger was unexpectedly not loaded in catch trials of the UNLINKED-L and UNLINKED-R test series, respectively. Importantly, the normal force responses triggered in the finger that was unexpectedly not loaded were considerably stronger than the responses in a finger that was primarily not loaded. A comparison of the data for the corresponding UNLINKED test series in Figure 4, $A$ and $C$, and Figure $1, A$ and $C$, clearly illustrate this point. Accordingly, the amplitude of the first force rate peak of responses elicited in a nonloaded finger depended on the behavior of the apparatus $\left(F_{(2,12)}=10.9 ; p<0.01\right)$, whereas it did not reliably influence the response onset latency (Fig. $4 B, D$ ). For the UNLINKED-L and UNLINKED-R test series, the interdigital reflex response expressed by the first force rate peak was stronger for a finger typically loaded than for the accompanying finger predominantly not loaded, i.e., the right and left finger, respectively. The behavior of the apparatus also influenced the proportion of trials in which a reliable normal force response was detected in a nonloaded finger $\left(F_{(2,12)}=59.1 ; p<0.01\right)$. The responsiveness varied with the response intensity of the force rate peak, but it always averaged above $\sim 65 \%$; the responsiveness of a loaded finger was always $100 \%$.

\section{Unpredictable behavior of the apparatus}

During the UNLINKED-LR test series, only the left or only the right finger was loaded with equal probability; during the excep- 


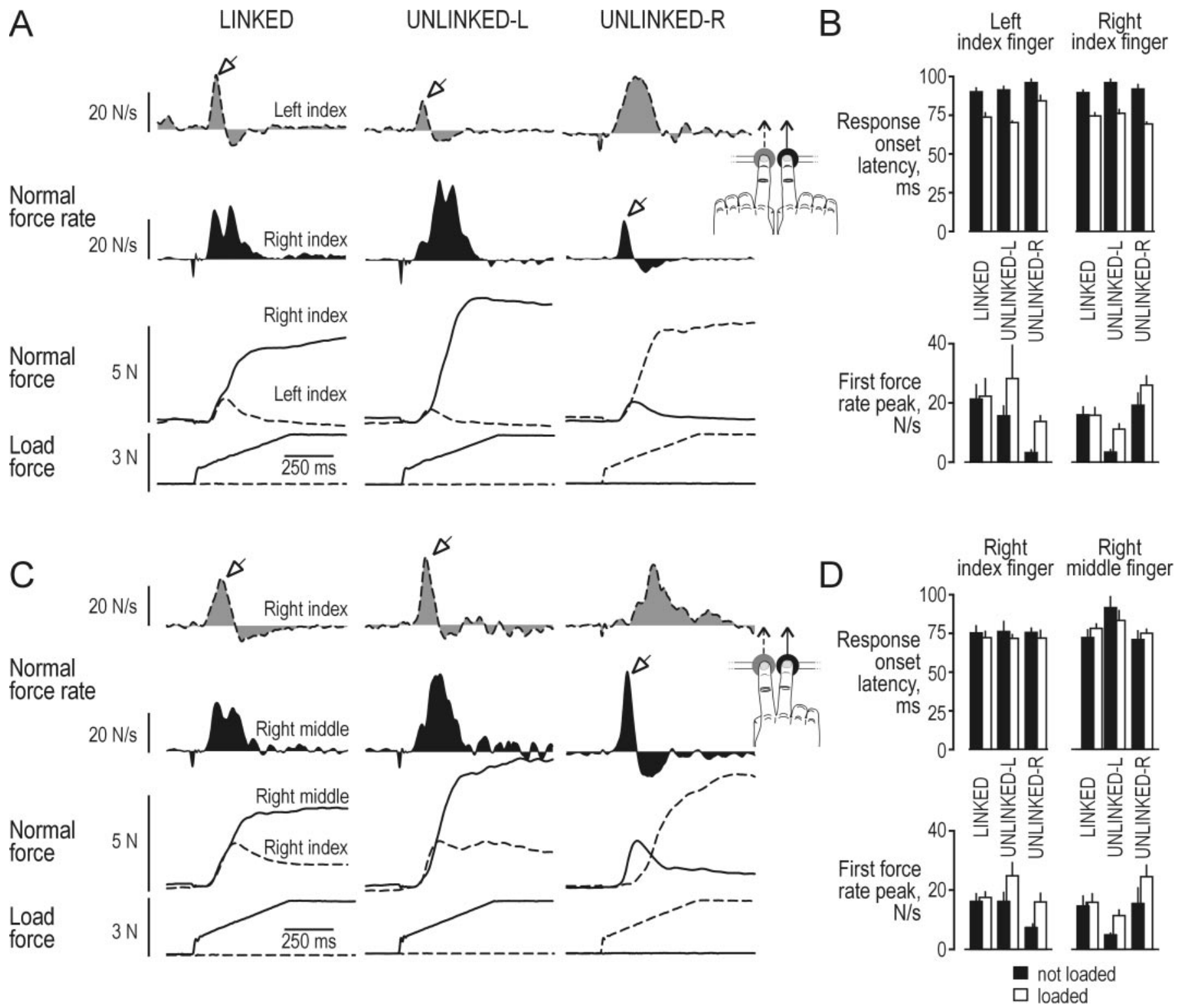

Figure 4. Responses in fingers unexpectedly not loaded. $A, C$, Data from the LINKED test series represent averaged responses in a single subject to loading of the right contact plate only. Data from the UNLINKED-L and UNLINKED-R represent responses when the left and right contact plate, respectively, was unexpectedly not loaded. Arrows indicate responses in fingers that were unexpectedly not loaded. For additional explanation, see caption of Figure 1, $A$ and $C$. B, D, Height of bars gives mean values for onset latency and first force rate peak for data averaged across all subjects; error bars indicate unilaterally one SEM $(n=7)$. Filled bars represent responses in a finger not loaded, and open bars represent, for comparison, responses in the same finger when loaded in isolation. $A, B$, Bimanual grasp. $C, D$, Unimanual grasp.

tional catch trials, both fingers were loaded concurrently. Longterm prediction of which finger was most likely to be loaded was impossible in these test series because the contact plates were loaded in a random order. However, it was conceivable that the subjects would use "short-term" predictions by preparing a response suitable for the loading condition in the last trials.

Figure 5 shows the responses to concurrent loading of the two contact plates in the UNLINKED-LR test series. The superimposed single trials are presented in groups with identical loading conditions in the previous trial, i.e., all trials were preceded either by a trial with loading of the left plate $(A, C)$ or the right plate $(B$, $D)$. In the bimanual test series, in particular when the right index finger had been loaded in the previous trial (Fig. 5B), concurrent loading often evoked normal force responses with similar temporal profiles at both fingers, regardless of the loading in the previ- ous trial. This suggests that the response during the bimanual grasp could be prepared for loading of whichever plate. In contrast, during the unimanual test series, the subjects primarily responded with a normal force response at either the left or the right finger. That is, they rarely responded synchronously at the two fingers but depended on corrective actions for one of the digits when the loading of the plates were linked. Apparently, the response preparation toggled between loading of one or the other finger. Hence, the finger control appeared more individuated during the unimanual than during the bimanual grasp configuration; the subsequent section further addresses differences between the two grasp configurations. Moreover, in neither the unimanual nor the bimanual grasps did the responses show any signs of predictions depending on the loading condition in the immediate previous trials. If the loading pattern of the previous 

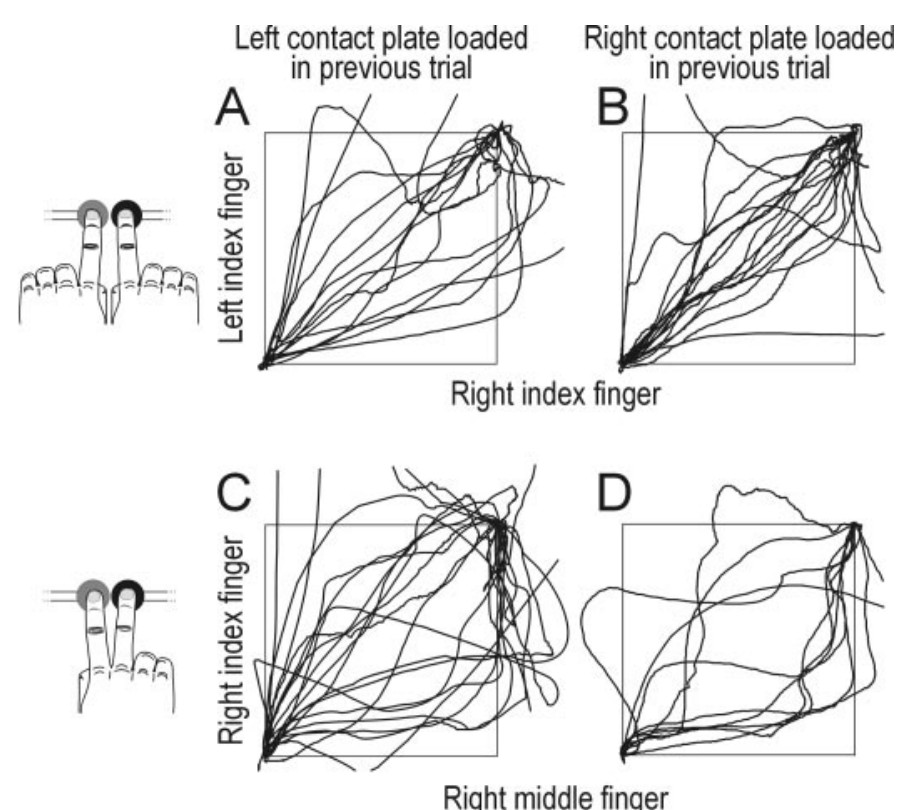

Figure 5. Responses when the behavior of the apparatus was unpredictable. Superimposed phase plots from single catch trials with concurrent loading of the fingers in the UNLINKED-LR test series; in these test series, typically only the left or only the right contact plate was loaded. Normal force at the finger contacting the left plate is plotted against the normal force at the finger contacting the right plate; forces were normalized as in Figure 2, $A$ and $C$. All applicable trials by all subjects were grouped by the loading condition of the preceding trial, i.e., loading of the left plate $(A, C)$ and the right plate $(B, D)$. In no case did the loading condition in the previous trial predict robustly which finger that would lead the force development, i.e., there were no clear signs of single-trial learning. $A, B$, Bimanual grasp. $C, D$, Unimanual grasp.

trials would have formed predictions in the UNLINKED-LR series, the phase plots should have looked like those of the UNLINKED-L (and UNLINKED-R) series (Fig. 2A,C) for trials preceded by loading of the left (and right) plate (Fig. $5 A, C$ ). That is, the finger loaded in the preceding trial would have led the normal force increase. However, there was no obvious relationship between the finger that lead the force development and whether or not that finger was loaded in the previous trial. Thus, our data did not provide any evidence for short-term predictions in this sense.

In the UNLINKED-LR test series, the response amplitude of a loaded finger was similar for the two fingers and was similar to that observed in the LINKED series. In contrast, the interdigital reflex interaction, indicated by the amplitude of first force rate peak of the normal force response of a nonloaded finger, was on average smaller than that during the LINKED series $\left(F_{(1,6)}=\right.$ $14.3 ; p<0.01)$ and corresponded to that which was observed for an infrequently loaded finger during the other UNLINKED series (Fig. 1 $A, C$ ).

\section{Differences between unimanual and bimanual grasp configurations}

Because of mechanical coupling of adjacent fingers by multitendoned muscles, we expected that the performance during the unimanual grasp would show subtler signs of individuated finger responses than that in the bimanual grasp. We found, however, great similarities between the two grasps. Specifically, the two grasps showed a similar capacity of response preparation, reflecting prediction of the behavior of the apparatus, and peripheral mechanical factors did not prevent a fractionated force output during the unimanual grasp. In fact, in several respects, a more fractionated behavior was observed during the unimanual than in the bimanual task. In the following, we describe differences that we observed between the bimanual and unimanual grasps concerning response preparation and corrective responses.

\section{Responses during linked trials}

For all types of behavior of the apparatus, subjects showed a similar capacity of response preparation and of execution of fractionated reactive finger forces during the two grasps. Furthermore, in the UNLINKED-LR test series, the subjects more often prepared for loading of one or the either of the contact plates during the unimanual grasp (Fig. 5).

The absolute difference of half-normal force latencies between cooperating fingers in linked trials was influenced by the grasp configuration $\left(F_{(3,18)}=18.7 ; p<0.01\right)$. In the LINKED test series, the difference was $22 \pm 8$ and $35 \pm 22 \mathrm{msec}$ during the bimanual and unimanual grasp, respectively. Thus, subjects showed a more synchronized effective force response during the bimanual than in the unimanual task. The corresponding values for the catch trails (linked trials) during the UNLINKED-L and UNLINKED-R were $98 \pm 49$ and $132 \pm 34 \mathrm{msec}$, and $78 \pm 43$ and $140 \pm 73 \mathrm{msec}$ for the UNLIKED-LR test series. Likewise, for trials selected for analysis of overt corrective responses, the latency of the corrective response $\left(F_{(1,4)}=24.6 ; p<0.01\right)$ was influenced by grasp configuration. The latency was $60 \pm 27 \mathrm{msec}$ longer for fingers engaged in the unimanual grasp compared with those engaged in the bimanual grasp. Thus, the corrective actions compensating for an erroneous prediction of the behavior of the apparatus appeared faster during the bimanual grasp compared with the unimanual grasp. A mechanical coupling of the digits through multitendoned muscles in the unimanual grasp can hardly explain this difference.

\section{Response onset latencies}

The grasp configuration influenced the response onset latency of a nonloaded finger $\left(F_{(1,6)}=25.7 ; p<0.01\right)$. In the unimanual grasp, the response onset of a nonloaded finger was similar to that of a loaded finger, whereas during the bimanual grasp, it occurred $\sim 15 \mathrm{msec}$ after that of the loaded partner finger (Ohki and Johansson, 1999). There was no effect by the behavior of the apparatus in this respect (Fig. 4, compare $B, D$ ).

\section{Pretrial normal forces}

It is known that one mode of response preparation in reactive grip tasks during loading at unpredictable times is to regulate the pretrial normal force (Johansson and Westling, 1988b; Johansson et al., 1992b; Cole and Johansson, 1993; Serrien et al., 1999; Winstein et al., 1999). In the present study, the behavior of the apparatus influenced the pretrial normal forces $\left(F_{(3,18)}=3.3 ; p<\right.$ 0.05 ; main effect), but this factor interacted with that of the grasp $\left(F_{(3,18)}=3.5 ; p<0.05\right)$. The behavior of the apparatus influenced the pretrial normal forces only during the unimanual grasp but only for the finger predominantly loaded in the UNLINKED-L and UNLINKED-R test series; in test series in which a finger was predominantly loaded in isolation, subjects exerted stronger pretrial forces at that finger than in any other test series $(p<0.05$ and $p<0.01$ for the index and middle finger, respectively; Tukey's honestly significant difference test). Thus, this response preparation represented fractionated finger actions. The magnitude of these influences was modest $(0.6 \mathrm{~N}$ for the right index and 
1.2 $\mathrm{N}$ for the right middle finger), and they should have at most marginally influenced the triggered normal force responses (Cole and Johansson, 1993; Winstein et al., 1999).

\section{DISCUSSION}

We conclude that the reflex mechanisms that modulate reactive responses at individual digits to changes in fingertip loads are under predictive control. Specifically, the automatic responses to sudden tangential load changes reflected whether or not the plates contacted by the two fingers were mechanically linked, so that the apparatus simulated a single object, or unlinked, so that the apparatus simulated two independent objects. As such, unexpected loading of one of the digits engaged in the task resulted in an initially weaker and delayed response, whereas significant early responses occurred in a digit that was unexpectedly not loaded. Reactive responses to load changes that challenge grasp stability at the level of individual digits are thus controlled in a manner akin to what has been demonstrated for the two hands engaged in proactive, bimanual motor behaviors (Blakemore et al., 1998)

The control of purposeful motor behaviors requires that subjects are able to predict the consequences of the generated actions. The basis for this is internal representations of both the expected sensory consequences of the actions ("corollary discharge;" Sperry, 1950) and the motor commands ("efference copy;" von Holst, 1954). Indeed, forward internal models that predict sensory consequences from efference copies of self-issued motor commands are considered critical for motor planning, execution, and learning (Jordan, 1995; Miall and Wolpert, 1996; Wolpert, 1997; Wolpert and Kawato, 1998; Kawato, 1999). In proactive manipulative tasks, the essential internal models are not limited the subject's own motor apparatus but incorporate critical predictable properties of the object(s) (Johansson and Cole, 1992; Lacquaniti, 1992; Massion, 1992). In the current study, we demonstrated that subjects predicted the properties of the objects and thus entertained forward models of tasks, although the tasks were not self-paced but driven by reflex mechanisms. In short, the onset of the activity of the object triggered the CNS to release motor commands directed to individual digits and adequate for the predominant behavior of the apparatus. If somatosensory information obtained early during the loading indicated a mismatch between the predicted and the actual behavior of the apparatus, the motor commands were corrected after some delay ( $\sim 100 \mathrm{msec}$ ) [cf. Johansson and Cole, 1994; Johansson, 1998 ("discrete-event driven control policy")]. This implies that subjects during manipulation generate internal representations of expected sensory consequences not only of their own actions but also of the actions imposed by external objects. Humans thus seem to be able to internalize and represent the effects of active objects in terms of appropriate motor behaviors and sensory consequences, similar to their innate capacity to internalize and represent the actions of other humans ("perception of action;" Meltzoff, 1990; Rizzolatti et al., 1996; Buccino et al., 2001).

The comparatively long neuromechanical delays in humans limit the usefulness of closed feedback control in motor control and promote control based on predictions (Rack, 1981; Hogan et al., 1987). We do not know how object properties such as weight, surface friction, or moment of inertia are represented in the CNS, yet humans easily and correctly predict the forces required to lift visually presented, familiar objects (Gordon et al., 1993). Moreover, normal humans learn in single trials to predict, for instance, the weight of an object (Johansson and Westling, 1988a) and mass distribution of the object rendering torques tangential to the grasped surfaces that challenge grasp stability in manipulatory maneuvers (Goodwin et al., 1998; Wing and Lederman 1998; Johansson et al., 1999). The amount of information available about natural objects with which humans can interact varies considerably. At one extreme, we may consider interactions with familiar, predictable, passive objects that, when manipulated, will give rise to destabilizing forces that are completely determined by the subject's own action (Johansson and Cole, 1994). The opposite extreme would be an object with few known properties and unknown future behavior. The task that faced the subjects in the current study should be considered within this spectrum of tasks. Interestingly, in the UNLINKED-LR test series during which no meaningful prediction was possible except that either one or the other finger would be loaded in isolation, subjects had great difficulties in releasing concurrent activity of the two fingers, especially in the unimanual grasp configuration (compare with Fig. 5). Apparently, the subjects primarily selected one or the other of the feedforward models appropriate for the behavior of the apparatus (cf. Flanagan et al., 1999; Wolpert and Kawato, 1998) [cf. Horak and Nashner, 1986 (selection of hip vs ankle strategy during stance perturbations)].

The response initiation and specification represent sensorimotor processes that can be dissociated as has been demonstrated in tasks involving reactive isometric elbow flexion (Ghez et al., 1989), automatic postural responses (Horak et al., 1989), and grip responses to unpredictably timed loads (Häger-Ross et al., 1996; Winstein et al., 2000). The present study provides yet another example of this. The influence of the behavior of the object on the response onset was rather scant (maximum latency effect of $\sim 7$ msec) [cf. Häger-Ross et al., 1996 (10 msec)]. In contrast, the predominant behavior of the apparatus markedly influenced the functionally more important subsequent response. During catch trials with concurrent loading of the digits in the predictable UNLINKED test series, for instance, the major force development at the infrequently loaded partner finger was delayed by $\sim 100$ msec compared with the finger that was predominantly loaded. Apparently reflecting the prevailing "sensorimotor set," subjects initially released motor commands specified for the predicted behavior of the apparatus and later, if necessary, made corrections.

When subjects lift stable and predictable objects, they efficiently adapt to object features critical for grasp stability in essentially a single trial. This has, for instance, been demonstrated with regard to surface friction (Johansson and Westling, 1984), weight (Johansson and Westling, 1988a), and object shape (Jenmalm and Johansson, 1997; Jenmalm et al., 2000). Although subjects adjusted to the properties of the manipulated object also in the present study, this adjustment was evidently not based on "singletrial" learning. In particular, although the overall behavior of the apparatus determined the response pattern, the loading pattern in a particular trial did not influence markedly the sensorimotor set in the immediately subsequent trial. It remains to be investigated whether learning correct predictions in our reactive tasks have some resemblance with that found by Witney et al. (2000) during proactive manipulation of linked and unlinked objects in bimanual tasks. If so, learning to predict loading would be quicker than learning to predict absence of loading at the level of individual digits. Moreover, the degree of temporal synchronicity of the loading of digits engaged in a task is likely to influence how easy it is to learn the active properties of an object (Witney et al., 1999). It remains to be established to which degree verbal de- 
scriptions of the physical properties of an object help development of predictions for reactive control.

Our observations that predictions of object properties affect the selection of reactive response patterns resemble observations of responses to support perturbations during stance (Horak and Nashner, 1986) and of reactive forearm movements evoked by perturbing a handheld bar. In particular, the behavior of the object seems to influence the late dynamic response more than the response initiation per se. With forearm movements, subjects' preparations influence neural activities in primary sensorimotor areas (Evarts and Tanji, 1974, 1976), and set-related neural activity change is observed in premotor (Godschalk et al., 1981; di Pellegrino and Wise, 1991; Riehle and Requin, 1993) and prefrontal cortices (Kubota and Funahashi, 1982; Watanabe, 1986; di Pellegrino and Wise, 1993). Interestingly, neurons in the prefrontal cortex increased the set-related activities proportional to the probability of the response they encode (Quintana and Fuster, 1992), and brain imaging has revealed activation in the corresponding areas when humans prepare self-paced movements (Roland, 1985; Deiber et al., 1996). There is evidence that reactive digital responses supporting grasp stability in humans involve both subcortical and cortical components (Johansson et al., 1992b, 1994; Harrison et al., 2000).

This study confirms findings in a previous study that the response at a nonloaded finger is essentially confined to an early force rate pulse in a bimanual grasp, whereas in a unimanual grasp, the amplitude of the response phases after this initial pulse are stronger and included a reliable static component (Ohki and Johansson, 1999). Whereas movements at individual digits can be achieved by a balanced activity of several muscles (Schieber, 1995), these findings could be explained by mechanical constraints between the index and the middle fingers attributable to multitendoned muscles (cf. Kilbreath and Gandevia, 1994) rather than by neural mechanisms. The present results revealed, however, that the purely mechanical effect must be minor. First, decreasing the probability of linkage of the two contact plates substantially attenuated the strength of the interactions compared with what was observed in the predominantly LINKED test series. Second, the unimanual grasp showed, if anything, a more fractionated grasp behavior than that of the bimanual grasp, and asynchronous responses at the partner fingers was more common and the asynchronousness was stronger in the unimanual than in the bimanual grasp configuration.

\section{REFERENCES}

Birznieks I, Burstedt MK, Edin BB, Johansson RS (1998) Mechanisms for force adjustments to unpredictable frictional changes at individual digits during two-fingered manipulation. J Neurophysiol 80:1989-2002.

Blakemore SJ, Goodbody SJ, Wolpert DM (1998) Predicting the consequences of our own actions: the role of sensorimotor context estimation. J Neurosci 18:7511-7518.

Buccino G, Binkofski F, Fink GR, Fadiga L, Fogassi L, Gallese V, Seitz RJ, Zilles K, Rizzolatti G, Freund H-J (2001) Action observation activates premotor and parietal areas in a somatotopic manner: an fMRI study. Eur J Neurosci 13:400-404.

Burstedt MK, Edin BB, Johansson RS (1997) Coordination of fingertip forces during human manipulation can emerge from independent neural networks controlling each engaged digit. Exp Brain Res 117:67-79.

Burstedt MK, Flanagan JR, Johansson RS (1999) Control of grasp stability in humans under different frictional conditions during multidigit manipulation. J Neurophysiol 82:2393-2405.

Cole KJ, Abbs JH (1988) Grip force adjustments evoked by load force perturbations of a grasped object. J Neurophysiol 60:1513-1522.

Cole KJ, Johansson RS (1993) Friction at the digit-object interface scales the sensorimotor transformation for grip responses to pulling loads. Exp Brain Res 95:523-532.

Deiber MP, Ibanez V, Sadato N, Hallett M (1996) Cerebral structures participating in motor preparation in humans: a positron emission tomography study. J Neurophysiol 75:233-247.

di Pellegrino G, Wise SP (1991) A neurophysiological comparison of three distinct regions of the primate frontal lobe. Brain 114:951-978.

di Pellegrino G, Wise SP (1993) Visuospatial versus visuomotor activity in the premotor and prefrontal cortex of a primate. J Neurosci 13:1227-1243.

Edin BB, Westling G, Johansson RS (1992) Independent control of human finger-tip forces at individual digits during precision lifting. J Physiol (Lond) 450:547-564.

Evarts EV, Tanji J (1974) Gating of motor cortex reflexes by prior instruction. Brain Res 71:479-494.

Evarts EV, Tanji J (1976) Reflex and intended responses in motor cortex pyramidal tract neurons of monkey. J Neurophysiol 39:1069-1080.

Flanagan JR, Tresilian J, Wing AM (1993) Coupling of grip force and load force during arm movements with grasped objects. Neurosci Lett 152:53-56.

Flanagan JR, Tresilian JR (1994) Grip-load force coupling: a general control strategy for transporting objects. J Exp Psychol Hum Percept Perform 20:944-957.

Flanagan JR, Wing AM (1997) The role of internal models in motion planning and control: evidence from grip force adjustments during movements of hand-held loads. J Neurosci 17:1519-1528.

Flanagan JR, Nakano E, Imamizu H, Osu R, Yoshioka T, Kawato M (1999) Composition and decomposition of internal models in motor learning under altered kinematic and dynamic environments. J Neurosci 19:RC34(1-5).

Ghez C, Hening W, Favilla M (1989) Gradual specification of response amplitude in human tracking performance. Brain Behav Evol 33:69-74

Godschalk M, Lemon RN, Nijs HG, Kuypers HG (1981) Behaviour of neurons in monkey peri-arcuate and precentral cortex before and during visually guided arm and hand movements. Exp Brain Res 44:113-116.

Goodwin AW, Jenmalm P, Johansson RS (1998) Control of grip force when tilting objects: effect of curvature of grasped surfaces and applied tangential torque. J Neurosci 18:10724-10734.

Gordon AM, Westling G, Cole KJ, Johansson RS (1993) Memory representations underlying motor commands used during manipulation of common and novel objects. J Neurophysiol 69:1789-1796.

Häger-Ross C, Cole KJ, Johansson RS (1996) Grip-force responses to unanticipated object loading: load direction reveals body- and gravityreferenced intrinsic task variables. Exp Brain Res 110:142-150.

Harrison LM, Mayston MJ, Johansson RS (2000) Reactive control of precision grip does not depend on fast transcortical reflex pathways in X-linked Kallmann subjects. J Physiol (Lond) 527:641-652.

Hogan N, Bizzi E, Mussa-Ivaldi FA, Flash T (1987) Controlling multijoint motor behavior. Exerc Sport Sci Rev 15:153-190.

Horak FB, Nashner LM (1986) Central programming of postural movements: adaptation to altered support-surface configurations. J Neurophysiol 55:1369-1381.

Horak FB, Diener HC, Nashner LM (1989) Influence of central set on human postural responses. J Neurophysiol 62:841-853.

Jenmalm P, Johansson RS (1997) Visual and somatosensory information about object shape control manipulative fingertip forces. J Neurosci 17:4486-4499.

Jenmalm P, Dahlstedt S, Johansson RS (2000) Visual and tactile information about object-curvature control fingertip forces and grasp kinematics in human dexterous manipulation. J Neurophysiol 84:2984-2997.

Johansson RS (1996) Sensory control of dexterous manipulation in humans. In: Hand and brain: the neurophysiology and psychology of hand movements. (Wing AM, Haggard P, Flanagan JR, eds), pp 381-414. San Diego: Academic.

Johansson RS (1998) Sensory input and control of grip. Novartis Found Symp 218:45-59.

Johansson RS, Cole KJ (1992) Sensory-motor coordination during grasping and manipulative actions. Curr Opin Neurobiol 2:815-823.

Johansson RS, Cole KJ (1994) Grasp stability during manipulative actions. Can J Physiol Pharmacol 72:511-524.

Johansson RS, Westling G (1984) Roles of glabrous skin receptors and sensorimotor memory in automatic control of precision grip when lifting rougher or more slippery objects. Exp Brain Res 56:550-564.

Johansson RS, Westling G (1988a) Coordinated isometric muscle commands adequately and erroneously programmed for the weight during lifting task with precision grip. Exp Brain Res 71:59-71.

Johansson RS, Westling G (1988b) Programmed and triggered actions to rapid load changes during precision grip. Exp Brain Res 71:72-86.

Johansson RS, Riso R, Häger-Ross C, Backstrom L (1992a) Somatosensory control of precision grip during unpredictable pulling loads. I. Changes in load force amplitude. Exp Brain Res 89:181-191.

Johansson RS, Häger-Ross C, Riso R (1992b) Somatosensory control of precision grip during unpredictable pulling loads. II. Changes in load force rate. Exp Brain Res 89:192-203.

Johansson RS, Häger-Ross C, Bäckstrom L (1992c) Somatosensory con- 
trol of precision grip during unpredictable pulling loads. III. Impairments during digital anesthesia. Exp Brain Res 89:204-213.

Johansson RS, Lemon RN, Westling G (1994) Time-varying enhancement of human cortical excitability mediated by cutaneous inputs during precision grip. J Physiol (Lond) 481:761-775.

Johansson RS, Bäcklin JL, Burstedt MK (1999) Control of grasp stability during pronation and supination movements. Exp Brain Res 128:20-30.

Jordan MI (1995) Computational aspects of motor control and motor learning. In: Handbook of perception and action: motor skills, Vol 2 (Heuer H, Keele S, eds), pp 71-118. New York: Academic.

Kawato M (1999) Internal models for motor control and trajectory planning. Curr Opin Neurobiol 9:718-727.

Kilbreath SL, Gandevia SC (1994) Limited independent flexion of the thumb and fingers in human subjects. J Physiol (Lond) 479:487-497.

Kubota K, Funahashi S (1982) Direction-specific activities of dorsolateral prefrontal and motor cortex pyramidal tract neurons during visual tracking. J Neurophysiol 47:362-376.

Lacquaniti F (1992) Automatic control of limb movement and posture. Curr Opin Neurobiol 2:807-814.

Macefield VG, Hager-Ross C, Johansson RS (1996) Control of grip force during restraint of an object held between finger and thumb: responses of cutaneous afferents from the digits. Exp Brain Res 108:155-171.

Massion J (1992) Movement, posture and equilibrium: interaction and coordination. Prog Neurobiol 38:35-56.

Meltzoff AN (1990) Towards a developmental cognitive science. The implications of cross-modal matching and imitation for the development of representation and memory in infancy. Ann NY Acad Sci 608:1-31.

Miall RC, Wolpert DM (1996) Forward models for physiological motor control. Neural Networks 9:1265-1279.

Ohki Y, Johansson RS (1999) Sensorimotor interactions between pairs of fingers in bimanual and unimanual manipulative tasks. Exp Brain Res 127:43-53.

Quintana J, Fuster JM (1992) Mnemonic and predictive functions of cortical neurons in a memory task. NeuroReport 3:721-724.

Rack PMH (1981) Limitations of somatosensory feedback in control of posture and movement. In: Handbook of physiology, Sec 1, The nervous system (Brookhart JM, Mountcastle VB, eds), pp 229-256. Bethesda, MD: American Physiological Society.
Riehle A, Requin J (1993) The predictive value for performance speed of preparatory changes in neuronal activity of the monkey motor and premotor cortex. Behav Brain Res 53:35-49.

Rizzolatti G, Fadiga L, Gallese V, Fogassi L (1996) Premotor cortex and the recognition of motor actions. Brain Res Cogn Brain Res 3:131-141.

Roland PE (1985) Cortical organization of voluntary behavior in man. Hum Neurobiol 4:155-167.

Schieber MH (1995) Muscular production of individuated finger movements: the roles of extrinsic finger muscles. J Neurosci 15:284-297.

Serrien DJ, Kaluzny P, Wicki U, Wiesendanger M (1999) Grip force adjustments induced by predictable load perturbations during a manipulative task. Exp Brain Res 124:100-106.

Sperry RW (1950) Neural basis of the spontaneous optokinetic response produced by visual inversion. J Comp Physiol Psychol 43:482-489.

von Holst E (1954) Relations between the central nervous system and the peripheral organ. Br J Anim Behav 2:89-94.

Watanabe M (1986) Prefrontal unit activity during delayed conditional Go/No-Go discrimination in the monkey. I. Relation to the stimulus. Brain Res 382:1-14.

Wing AM (1996) Anticipatory control of grip force in rapid arm movements. In: Hand and brain: neurophysiology and psychology of hand movement (Wing AM, Haggard P, Flanagan JR, eds), pp 301-324. San Diego: Academic.

Wing AM, Lederman SJ (1998) Anticipating load torques produced by voluntary movements. J Exp Psychol Hum Percept Perform 24:1571-1581.

Winstein CJ, Merians AS, Sullivan KJ (1999) Motor learning after unilateral brain damage. Neuropsychologia 37:975-987.

Winstein CJ, Horak FB, Fisher BE (2000) Influence of central set on anticipatory and triggered grip-force adjustments. Exp Brain Res 130:298-308.

Witney AG, Goodbody SJ, Wolpert DM (1999) Predictive motor learning of temporal delays. J Neurophysiol 82:2039-2048.

Witney A, Goodbody SJ, Wolpert DM (2000) Learning and decay of prediction in object manipulation. J Neurophysiol 84:334-343.

Wolpert DM (1997) Computational approaches to motor control Trends Cogn Sci 1:209-216.

Wolpert DM, Kawato M (1998) Multiple paired forward and inverse models for motor control. Neural Networks 11:1317-1329. 